\title{
Interactive Implants: Vision and challenges
}

Klaus-Peter Hoffmann, Fraunhofer-Institut für Biomedizinische Technik, Sulzbach, Germany, klaus-peter.hoffmann@ibmt.fraunhofer.de Heidi, Olze, Klinik für Hals-, Nasen-, Ohrenheilkunde, Charité - Universitätsmedizin Berlin, Berlin, Germany, heidi.olze@charite.de

Werner Kneist, Klinik für Allgemein-, Vsizeral- und Transplantationschirurgie, Universitätsmedizin Mainz, Mainz, Germany, werner.kneist@unimedizin-mainz.de Rüdiger Rupp, Klinik für Paraplegiologie, Universitätsklinikum Heidelberg, Heidelberg, Germany, ruediger.rupp@med.uni-heidelberg.de

Thilo Krüger, inomed Medizintechnik GmbH, Emmendingen, Germany, T.Krueger@inomed.com Wiebke Droste, Universität Mannheim, Institut für Deutsches, Europäisches und Internationales Medizinrecht, Gesundheitsrecht und Bioethik der Universitäten Heidelberg und Mannheim (IMGB), Germany, wiebke.droste@imgb.de Marc Ruta, Wilddesign GmbH \& Co. KG, Gelsenkirchen, Germany, marc.ruta@wilddesign.de

Different complex physiological functions could be restored with distributed active implants. Wireless communication between the implants and an external control unit is necessary to synchronize the electrical stimulation of neural and muscular tissue. The recording of bioelectrical signals allows the implementation of closed loop stimulation regimes. In a semi-automated process, the network can be configured for and adapted to different applications and specific patient requirements. Depending on the actual needs, patients may adjust stimulation parameters on their own to individually optimize the therapy outcome.

Seventeen project partners representing industry, academia, healthcare providers and research organizations work together under umbrella of the BMBF Innovation Cluster "Interactive Implants (INTAKT)". The goal of this project cluster is to develop, characterize and use the implant network. Challenges in the project cluster include complex interaction between humans and technology, wireless power supply, long-term implant stability as well as secure and safe signal transmission. Functional demonstrators will be developed and validated for different applications:

(a) Tinnitus suppression using electrical stimulation

(b) Gastrointestinal electrical stimulation and

(c) Restoration of grasping function in paralyzed hands. 


\section{Tinnitus suppression using electrical stimulation}

Heidi Olze, Department of ORL, Head and Neck Surgery, Charité - Universitätsmedizin Berlin, Berlin, Germany, $\underline{\text { Hei- }}$ di.Olze@charite.de

Agnieszka J. Szczepek, Department of ORL, Head and Neck Surgery, Charité - Universitätsmedizin Berlin, Berlin, Germany, Agnes.Szczepek@charite.de / Uta Reich, Department of ORL, Head and Neck Surgery, Charité - Universitätsmedizin Berlin, Berlin, Germany, U.Reich@ charite.de / Stefan Gräbel Department of ORL, Head and Neck Surgery, Charité - Universitätsmedizin Berlin, Berlin, Germany, Stefan.Graebel@charite.de / Florian Uecker, Department of ORL, Head and Neck Surgery, Charité - Universitätsmedizin Berlin, Berlin, Germany, Fc.Uecker@charite.de

Tinnitus is a phantom sound perceived only by the affected person. Although the causes for tinnitus may be very diverse (e.g. hearing loss, cardiovascular diseases, diabetes, Meniere's disease, otosclerosis), they all lead to pathological activation of the auditory pathway.

Cochlear implants are electronic inner ear prostheses used to restore the hearing abilities of hard of hearing people. It has been noticed that the cochlear implants not only enable hearing but also reduce tinnitus that usually accompanies deafness and hearing loss. One of the likely mechanisms behind the latter phenomena is thought to be the unknown properties of electrical stimulus.

This project was designed as a proof of concept for a device, which would be sending the tinnitus-suppressing electrical signal. To achieve this, the project was split into a preclinical part using experimental animals (guinea pigs) and a clinical trial part with tinnitus patients. In the preclinical part, together with Inomed Medizintechnik $\mathrm{GmbH}$, we are testing the physical design and biocompatibility of anti-tinnitus implant. In the clinical part of the project, we use an electrical stimulation in the ear canal, through the eardrum (tinnitus patients), or via cochlear implant in a sample of cochlear implant patients (pre- and post-implantation). We ask the patients to mark the loudness of their tinnitus on a visual scale from one to ten before, during and after routine electrical stimulation and quality of the tinnitus.

The results obtained during our preclinical and clinical testing as well as future plans will be presented and discussed. 


\section{Interactive Implants: Restoration of grasping function in individuals with high spinal cord injury}

Rüdiger Rupp, Heidelberg University Hospital, Spinal Cord Injury Center, Heidelberg, Germany, ruediger.rupp@med.uni-heidelberg.de

Andreas Kogut, Heidelberg University Hospital, Spinal Cord Injury Center, Heidelberg, Germany, andreas.kogut@med.uni-heidelberg.de

Marcel Böttrich, Technical University Ilmenau, Institute for Biomedical Engineering and Informatics, Ilmenau, Germany, marcel.boettrich@tu-ilmenau.de

Kai Diercks, Soventec GmbH, Dannewerk, Germany, kai.diercks@soventec.de

Roman Ruff, Fraunhofer Institute for Biomedical Engineering, St. Ingert, Germany, roman.ruff@ibmt.fraunhofer.de

Klaus-Peter Hoffmann, Fraunhofer Institute for Biomedical Engineering, St. Ingert, Germany, klauspeter.hoffmanmn@ibmt.fraunhofer.de

The bilateral loss of grasping as a consequence of a cervical spinal cord injury (SCI) negatively impacts the quality of life of the affected individuals and their ability to fully participate in life. If surgical methods (tendon transfers of muscles still under voluntary control) are not applicable, grasp neuroprosthesis on the basis of function electrical stimulation are the only option for improvement of an impaired hand function. Neuroprostheses using cutaneous stimulation electrodes have several limitations including poor selectivity, restricted reproducibility of only a few grasp patterns and handling problems. But even the formerly commercially available, implantable Freehand grasp neuroprostheses still had several disadvantages including joint-spanning cables (risk of mechanical breakdown, fast spread of infections), non-scalability to the neurological status of each individual user and a non-intuitive control by shoulder movements. Most of the problems of grasp neuroprostheses can be overcome by the distributed active implants developed in INTAKT. With up to 12 implants, the restoration of 6 different grasp patterns together with wrist roation is possible. The implant network will be powered by coils integrated into a textile forearm sleeve and will communicate with each other and with the external control unit by infrared and/or radio link. A crucial component of any neuroprosthesis is the man-machine interface. The end user of the INTAKT neuroprosthes will use AR-glasses with integrated eye-tracking / lid closing detection functionality for grasp pattern selection, grip force adjustments and to initiate an emergency stop. In more than $60 \%$ of individuals with cervical SCI, residual muscle functions are still present. In these cases, the INTAKT implants can be configured to record electromyographic activities allowing for intuive control. While the basic functionality of the user interface is currently tested with a noninvasive neuroprosthesis in human users, functional evaluation of the implant network will be performed in pigs. 


\title{
Electrical stimulation with motility analysis of five parts of the gastrointestinal tract
}

\section{First evaluation of an experimental protocol in a porcine model}

\author{
Jonas F. Schiemer*, Axel Heimann, Karin Somerlik-Fuchs, Roman Ruff, Klaus-Peter Hoffmann,
} Jan Baumgart, Werner Kneist, Johannes Gutenberg University Mainz, Germany

Gastrointestinal (GI) motility disorders are frequent and clinically significant conditions with impairment of patient's quality of life. Examples range from upper GI symptoms such as dysphagia and gastroparesis to lower GI manifestations, namely chronic-intestinal pseudo obstruction, diarrhea and constipation. Furthermore, postoperative motility disorders are common. Currently, available pharmacological or dietetic treatment options are limited. Since GI motility is based on myoelectric activity, electrical stimulation (ES) is a promising alternative. Numerous studies have demonstrated suitable pacing strategies and parameters in different GI segments. However, results of multilocular ES are rare. We report the first experimental study to evaluate ES of five GI parts in a porcine model. Multi-channel electromyography (EMG) recordings of gastrointestinal baseline and poststimulatory electrical activity were realized together with videobased marker tracking (VBMT). ES provoked visible GI contractions and appeared to modulate frequencies of slow waves and spikes. Further investigations are needed for analysis of locoregional and cross-organ effects of ES on the GI tract.

Keywords: Gastrointestinal motility, Motility disorders, Electromyography, Video analysis, Electrical stimulation 


\section{Interactive implants: Translation in a medical product}

Thilo B. Krüger, inomed Medizintechnik GmbH, Emmendingen, Germany, t.krueger@inomed.com Karin H. Somerlik-Fuchs, inomed Medizintechnik GmbH, Emmendingen, Germany, k.somerlik@inomed.com

The BMBF funded national innovation cluster INTAKT (FKZ: 16SV7644, www.intakt-projekt.de) will establish a distributed implant research platform for different applications. Applications are Tinnitus suppression using electrical stimulation, gastrointestinal electrical stimulation and restoration of grasping function in paralyzed hands. To translate a technical development into a medical product, one has first to decide an application area, as medical products have a designated intended use. Therefore, a generic CE mark for all application areas is impossible to reach. After design freeze in the development all tests for electrical safety and electromagnetic compatibility must be made, this is based on standards, mainly DIN EN 60601 and sub-standards are applicable, but also implant specific safety norms have to be considered. Additionally, biocompatibility testing according to DIN EN ISO 10993 for implants is necessary. Testing must be performed in an accredited test laboratory. Therefore, external test costs in several hundred thousand Euros apply. No technical documentation like requirements engineering, risk analysis or other is then achieved yet. Finally all files need to be submitted to a notified body, which must be accredited according to the medical device directive 90/385/EEC to approve active implantable medical devices. The overall process after design freeze of the technical development takes minimum two years, if no clinical study is required. Then the interactive implant is valid for usage as medical product. However, clinics and hospital will not implant costly products without reimbursement. For new methodical applications since 2016 in Germany the $\$ 137 \mathrm{~h}$ SGB applies, adding an additional hurdle for the translation. Not mentioned that the need of a clinical study, which is in all likelihood extremely costly, must be discussed. In summary the translation towards a refundable medical product is possible but includes high efforts in time and money, so additionally financing is needed. 


\section{Interactive Implants: Ethical, legal and social implications}

Ri'in AG Wiebke Droste, Wissenschaftliche Mitarbeiterin, Institut für Deutsches, Europäisches und Internationales Medizinrecht, Gesundheitsrecht und Bioethik der Universitäten Heidelberg und Mannheim (IMGB), Schloss/Postfach, 68161 Mannheim, wiebke.droste@imgb.de

The use of intelligent implants and prostheses offers significant advances for optimizing medical treatment methods and is combined with an increasing technological modification of human body. This raises many exciting questions concerning law, ethics and social implications: Does the use of these techniques stay in line with our legal system and if yes, can the technical use be limited by governmental restrictions under certain conditions? Have moral values to be incorporated into technical artifacts? If machines are recreated from human beings, do they have to be provided with a legal subjectivity?

Medical devices have to be compatible with a high level of protection of health and safety. Therefore challenges, which are consequences of the indeterminate behaviour and the autonomous learning abilities of artificial intelligence, have to be faced. For avoidance of any hazards for legally protected rights medical product manufacturers have to assume legal duties, which affect construction, instruction and product development. For medical professionals there are also liabilities of maintenance measures and technical control that have to be accomplished.

Associated with the use of intelligent medical devices is the processing of personal data on health. Health data have a high potential for misuse and discrimination and therefore they're in need of special protection, particularly with regard to the development of "Big Data", "Ubiquous computing", "Internet of Things" and constantly increasing cybercrime.

If damages are suffered from the use of intelligent medical devices, difficulties in determining infringing acts, causality and culpability occur, especially because of the missing view into the technical progress of decision-making. It has to be explored, if the current liability law is able to assign responsibility adequately, otherwise there has to be found an appropriate concept for liability. 